\title{
Sea Level Anomaly and Earthquake Predictions: Endangered Countries Prognostications
}

\author{
Raini Hassan, Abid Ebna Saif Utsha, Mahfuzealahi Noman \\ Department of Computer Science, Kulliyyah of ICT, International Islamic University Malaysia, Gombak, Malaysia, \\ hrai@iium.edu.my, abidebnasaifutsha@gmail.com, noman.alahi@gmail.com
}

\begin{abstract}
Natural calamities are often unforeseen and cause massive destruction. It is extremely difficult to predict natural disasters. Existing machine learning techniques are not reliable enough to find the affected countries due to earthquakes and rising sea levels. This paper aims to use predictive analysis to find the countries that will be affected by earthquakes and rising sea levels. Also, the purpose is to see how machine learning techniques perform in terms of sudden calamities like earthquakes or slow calamities like rising sea level. The results were deduced by data analysis, and deep learning techniques like Long-Short Term Memory (LSTM). It was found out that using the approached method in this paper can accurately identify the countries that are going to be affected and predict both earthquake and sea level anomalies accurately. For earthquake, the model was able to capture the happening of earthquake events into a certain quarter of the year with the Root Mean Square Error (RMSE) of 0.504. And for sea level rise, the RMSE was 0.064. It was concluded that Deep learning techniques (e.g.-LSTM) work well with slow changes like sea level anomaly rather than sudden calamities like earthquakes. The techniques used in this paper can be upgraded further in the future to find and help more endangered countries to be prepared better against these natural calamities.
\end{abstract}

Keywords - earthquake prediction, sea level anomaly detection, deep learning, predictive analysis, LSTM

\section{INTRODUCTION}

Earthquakes can cause havoc in a region with its massive destruction. One such example would be the earthquake in Minahasa Peninsula, Indonesia occurred in the year 2018, the magnitude of the earthquake was 7.5 and the death toll was around 4,300 [1]. This earthquake also caused a Tsunami with a range of 7 to 15 meters. Before that Indonesia was hit by another 6.9 magnitude earthquake [2]. Most of the major earthquakes under the seabeds can cause massive Tsunamis that destroy lives and properties in the coastal region. It is impossible to predict an earthquake for humans. The scientists have found out Common toads (Bufo bufo) can detect impending seismic activity and alter their behavior [3]. While more studies are being done in that area, alternative effective solutions are needed quickly. The countries can prepare better if a system was able to predict accurately the event before it is happening. Currently, Earthquake warning system is based on triggered seismic events, based on seismic Primary Waves, Seismologist can warn people that more earthquakes (Secondary Waves) are coming and save lives with those warnings [22].

Sea level Rising is becoming a global concern now. Around 60 percent of the world's population lives in coastal areas. Due to climate change, the sea level is rising overwhelmingly. Already some of the coastal countries are noticing the change. One such example of this is the Maldives, which will be sinking under the sea within 80 years [4]. Another example found was Venice, which is also facing a similar risk. Also, many coastal areas will face flooding more frequently as well as permanent land loss which will disrupt the livelihood of coastal citizens. Which in turn will see migration towards inland areas from coastal areas. This will have a serious negative impact on countries' infrastructure and public systems, the whole country may face disarray and chaos. With proper prediction, the countries will know exactly when their country is going to sink and take proper precautions and measurements.

\section{Previous Works}

Implementing machine learning with earth science is still relatively new. Some of the researchers concluded that earthquake is impossible to predict while others suggested that it can be predictable using historic earthquake records. Many researchers used Artificial Neural Network (ANN) for earthquake predictions [5]. Some others predicted whether the earthquake is going to happen in one of the most earthquake-prone regions [6] [10]. Some of the machine learning technique used in that research were Pattern Recognition Neural Network (PRNN), Recurrent Neural Network (RNN), Random Forest (RF), Linear Programming (LPBoost). While many other researchers were using deep learning techniques to detect an earthquake and identify earthquake locations [7], other researchers applied deep learning techniques for the aftershock pattern after a large earthquake [8]. Deep learning technique was also used to predict an earthquake. To increase the model performance, instead of a single Long-Short Term Memory (LSTM) 
network, the researcher decomposed the original LSTM into several small ones [9]. Some other techniques used by researchers were Support Vector Machines (SVM) for seismic wave detection [10], the combination of Generative Adversarial Network (GAN) and RF to recognize primary waves of the earthquake [11]. While some articles provided comparison and conclude that deep learning yielded better results in time series analysis than other existing traditional machine learning techniques [12].

Sea level anomaly is the difference between actual sea surface height and mean sea surface height. Very few works have been done on combining sea level anomaly prediction and machine learning. As founded by Gamboa in his research that detecting anomaly from time-series analysis is challenging [12]. Some scientists forecast sea level changes in the Caspian Sea using SVM and Gene Expression Programming (GEP) [13]. Deep learning technique was also used to predict sea level anomaly [14], the researchers focused northern and equatorial Pacific region and apply LSTM, Convolutional Neural Network (CNN) + LSTM (ConvLSTM), Sequence LSTM, Sequence LSTM-P deep learning technique. Other researchers developed and validated two machine learning approach to forecasting sea level anomaly, one of the techniques was Support Vector Regression (SVR) and the other was Autoregressive Integrated Moving Average (ARIMA) [15]. The research area was the Pacific Ocean which is highly vulnerable to sea level rise.

The main purpose of this research to identify the endangered countries based on earthquake and sea level anomaly predictions. The target is to find the countries that are facing high risk due to earthquake or sea level rising or due to both. From the previous works, it can be seen clearly that many researchers are moving towards deep learning techniques to predict both sea level anomaly and earthquakes. This research will use deep learning techniques Long-Short Term Memory (LSTM) for both earthquake and sea level anomaly prediction. Also, it is found that earthquake and sea level varies based on geographic position. In some areas, earthquake is more deadly and happening more often than in other areas. For sea level as well, coastal countries and low-level countries are more at risk than other hinterland areas. For the simplicity and demonstration of the work, this research will focus only on Indonesia for both earthquake and sea level anomaly prediction. And it will be shown later why Indonesia selected for both sea level anomaly and earthquake. Another aim of this research is to see whether the prediction will show good results in predicting sudden natural disasters like earthquakes or a slow and inevitable disaster like rising sea levels.

\section{Methodology}

In this section, the process of predicting earthquake and sea level anomaly is discussed in detail synchronously.

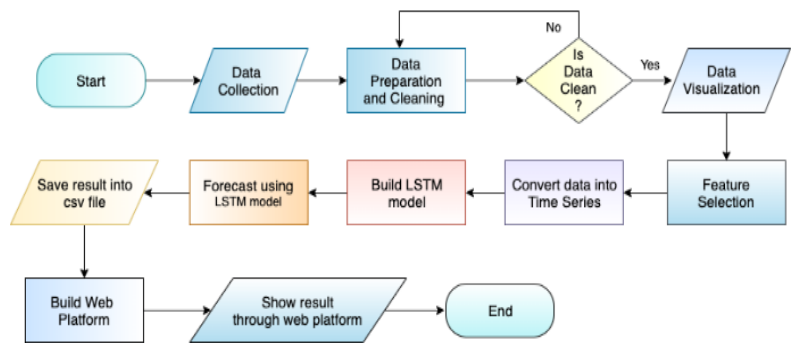

Fig. 1 The Workflow

\section{A. Data Collection}

The data was collected from various sources for both earthquake and sea level anomaly.

1) Earthquake: For earthquake predictions, the dataset was collected from the United States Geological Survey (USGS) earthquake catalog [16]. The records for the earthquake were from 1965 to 2019.

2) Sea Level Anomaly: Sea level anomaly dataset was collected from Essential Climate Variables (ECV) products of Climate Change Initiatives by the European Space Agency (ESA) [17] [18]. The records for the sea level anomaly was from 1993 to 2015. Another dataset was collected from World Bank which has records for population, land and other information regarding 84 coastal developing countries [19]. This dataset was collected to find out which countries will lose most land areas due to the rise of sea level.

\section{B. Data Pre-processing and Visualization}

1) Earthquake: The earthquake dataset was ranged into four categories depending on their magnitude range. Fig. 2 shows a quick visualization for the whole world to see the earthquake that happened over the years by category.

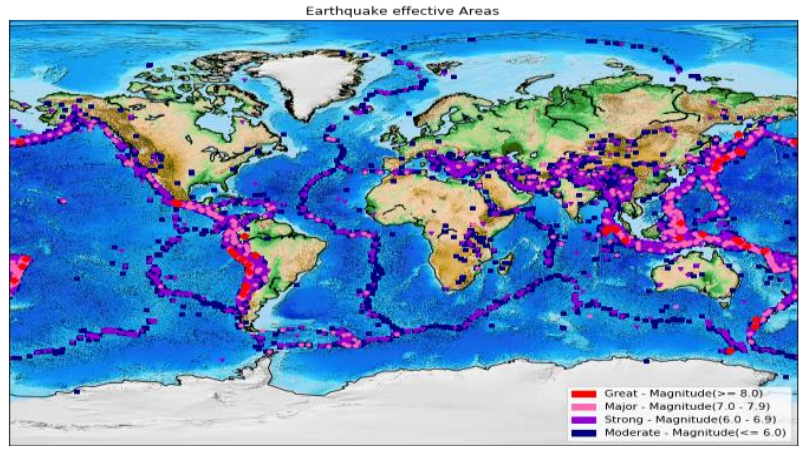

Fig. 1 Earthquake affected areas in the whole world by category 
The dataset was filtered for magnitude equal to 5.5 or above magnitude earthquake records only because normally earthquake magnitude below 5.5 has less impact compared to a magnitude equal or above 5.5. Then, a list was created from the earthquake data records to see which area had more earthquakes from 1980 to 2019. It was found out that Indonesia and its surroundings had around 1895 earthquakes within these years. The surrounding area like Java sea was also counted because it will have a direct impact of a tsunami if the earthquake magnitude is high. The experiment was conducted in Indonesia only. Only the necessary attribute for example year, month and others were selected. The dataset was transformed once again into four quarters in a year and in twelve months according to the date. The dataset later was split into training data which had 1705 records and testing data which had 190 records. Then the modified data was passed into the model building stage.

2) Sea Level Anomaly: The Sea level anomaly dataset was in NetCDF format. To verify and understand the NetCDF format better, panoply tool from the National Aeronautics and Space Administration (NASA) was used to visualize and see the attributes [20]. From Fig. 3 and Fig. 4 , it is clearly seen the changes in the sea level happened in the years 1993 and 2015.

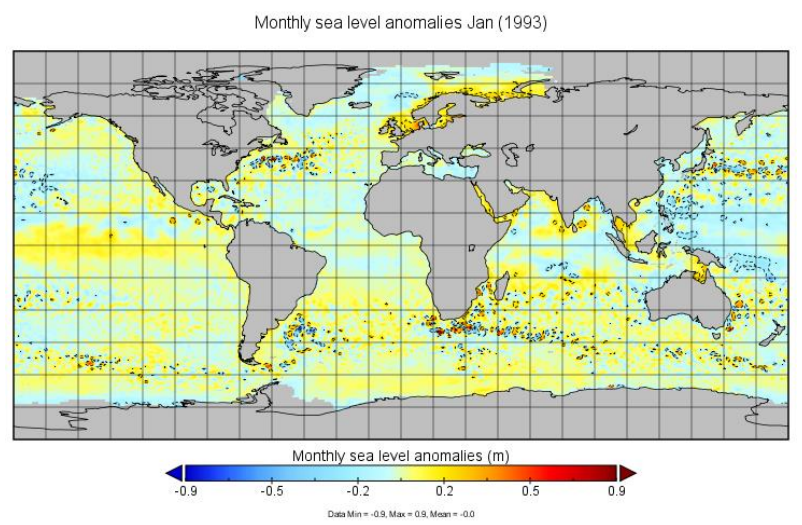

Fig. 3 Sea level anomalies in January 1993

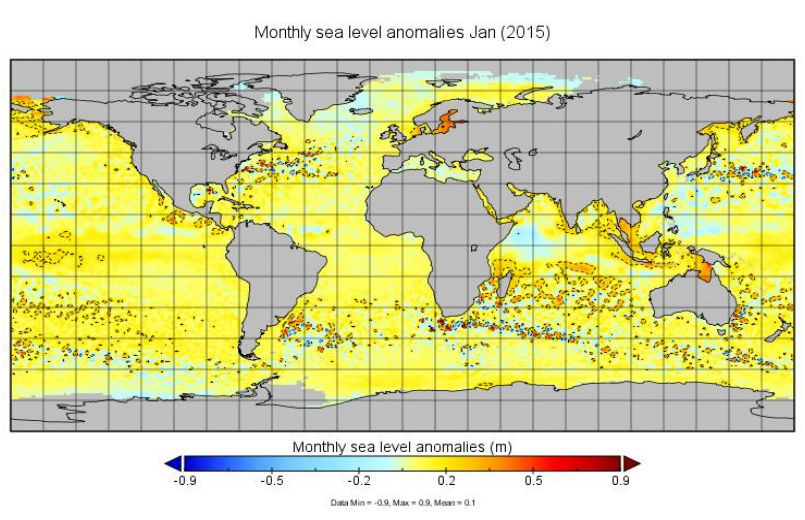

Fig. 4 Sea level anomalies in January 2015
In 1993 the sea level anomaly was mostly between $-0.5 \mathrm{~m}$ to $-0.2 \mathrm{~m}$ whereas in 2015 it became $0.1 \mathrm{~m}$ to $0.2 \mathrm{~m}$. The dataset was converted into a suitable format to conduct the model building. The raw NetCDF file had four dimension timeframe, latitude, longitude, sea level anomaly (in meter), For a range of latitude and longitude (Jakarta), timeframe and sea level anomaly was selected from raw data and converted into two-dimensional data. Also, the preprocessing was done to find out the most impacted countries according to the dataset [19]. There were many affected countries, Indonesia was selected because, in South-East Asia, it is one of the most vulnerable and affected countries. Then from the dataset points surrounding the city, Jakarta was selected and records from the year 1993 until 2015 were taken into consideration. The dataset was divided by training and test set. The training set was from the year 1993 until 2014 and the records of 2015 were test set.

\section{Model}

In this section, the proposed two-stacked LSTM model has been discussed. LSTM is an improvised version of traditional RNN and it can learn relations among data during a longtime interval. While training the network, Dropout helps to exclude activation and weight updates in input and recurrent connections to LSTM units which helps to reduce overfit and improve model performance. Additionally, in training data, 'Adam' optimization algorithm was used to update network weights iteratively instead of the classical stochastic gradient descent. Fig. 5 is showing a simple diagram of the implemented LSTM model.

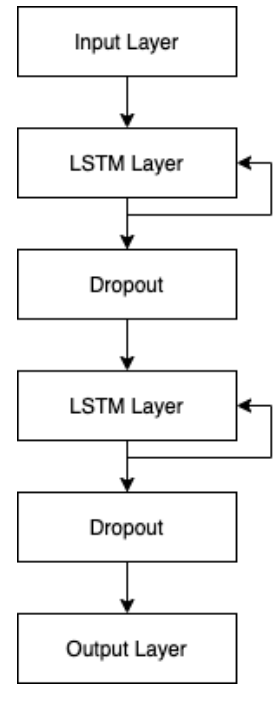

Fig. 5 The Flow diagram of the model

1) Earthquake: Deep learning technique Long Short Term Memory (LSTM) was considered for predicting the earthquake. The TensorFlow library with Keras was used for implementing the LSTM model and the whole process was 
run by GPU using Google Colab. Two-stacked LSTM model was used with two layers and a dropout technique. The first layer had 128 neurons and 0.2 dropout was applied. For the second layer, 256 neurons and 0.3 dropout were used. And 'Adam' optimizer was used while the loss was 'mean square error'. A total of 50 epochs and batch size 32 were applied to the model.

2) Sea Level Anomaly: The same architecture of earthquake model was followed here as well with the inclusion of the ARIMA model. ARIMA model performs well in forecasting future values for time series data. ARIMA model was used to forecast sea level anomaly along with LSTM model. LSTM model had the same architecture with two stacked layers and neurons. The optimizer and loss function were also same as well as the number of epochs and batch sizes.

\section{RESULTS AND ANALYSIS}

In this section, the results yielded from the model will be showed and analyzed for both earthquake and sea level anomaly.

\section{A) Earthquake}

The model tried to predict the upcoming earthquake and the time it will be happening. The time was broken into the year, a quarter of the year and month. After getting the results from the model, it was rounded into more suitable integer values. From Table 1, the model predicted an earthquake magnitude equal to or over 5.5 in Indonesia or surrounding Indonesia will happen in the year 2019 and into the fourth quarter of the year and in October.

Table 1

Earthquake predicted values

\begin{tabular}{|l|l|l|}
\hline Year & Quarter & Month \\
\hline 2019 & 3 & 9 \\
\hline 2019 & 3 & 9 \\
\hline 2019 & 3 & 9 \\
\hline 2019 & 3 & 9 \\
\hline 2019 & 3 & 9 \\
\hline 2019 & 3 & 9 \\
\hline 2019 & 3 & 9 \\
\hline 2019 & 3 & 9 \\
\hline 2019 & 4 & 11 \\
\hline 2019 & 4 & 10 \\
\hline 2019 & 4 & 10 \\
\hline
\end{tabular}

To evaluate the model, Root-Square Mean Error (RMSE) method was used. The result was also broken down further into a quarter of the year and month to identify the model's ability to recognize when the earthquake is going to occur. The RMSE for predicting the quarter of the year was 0.504 and predicting the month was 1.610 .

\section{B) Sea Level Anomaly}

First, the ARIMA model was conducted to predict sea level anomaly. The result of the ARIMA model shown in Fig. 6 . Then, the LSTM model was conducted, and the result is shown in Fig. 7. The blue line represented the predicted value, and the red line represents real value. The model is predicting that the sea level anomaly surrounding Jakarta would be approximately 0.05 in September in 2016. Also, to evaluate the model RMSE for sea level anomaly prediction was calculated and the value was 0.064 .

\section{DISCUSSION}

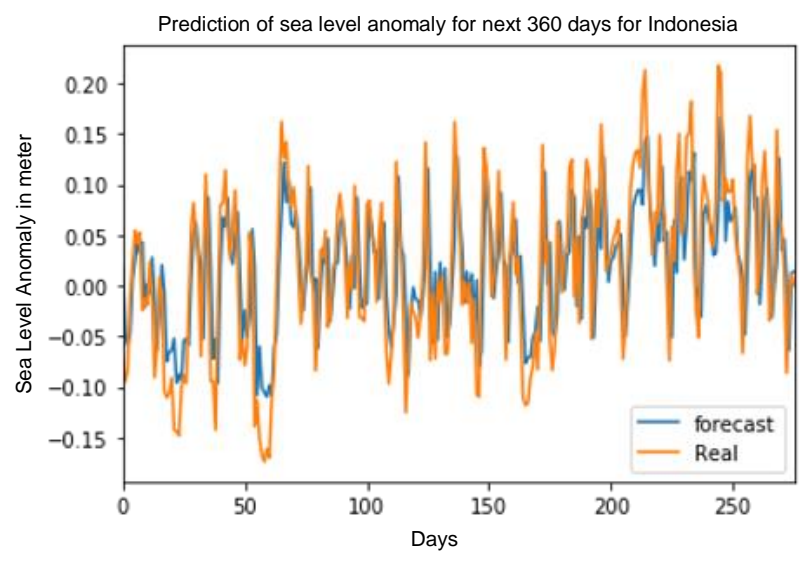

Fig. 6 The forecasting using ARIMA model

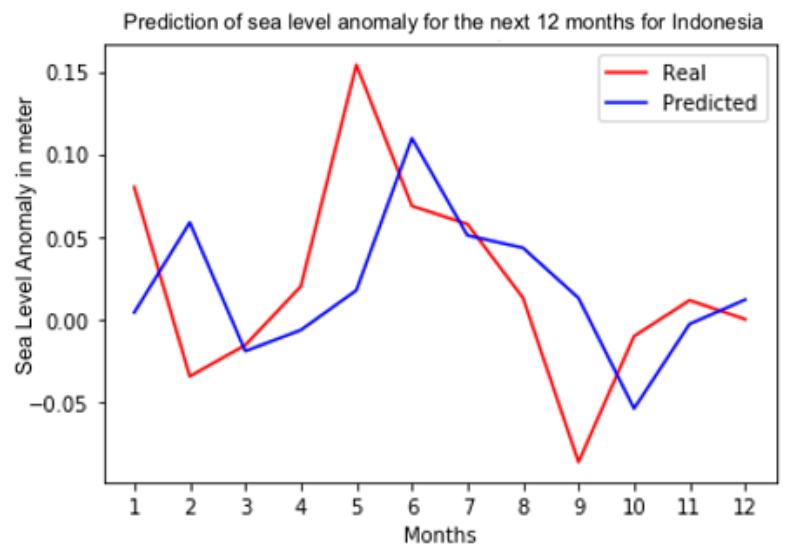

Fig. 7 The forecasting using LSTM model

In this section, further discussion on the results was done for both earthquake and sea level anomaly prediction.

\section{A) Earthquake}

From the result and analysis section, the model was able to identify the quarter of the month correctly and could not be able to identify the month accurately yet. The model can accurately identify the earthquake that will happen in the year 2019 and between October to December in Indonesia. The model also predicted the month of October but there was high RMSE in the model predicting for the month. To 
predict an earthquake with exact accuracy is a difficult task because there are many things related to the earthquake. One of the many related things is the movements of tectonic plates.

\section{B) Sea Level Anomaly}

From the result and analysis section, the model was able to predict sea level anomaly correctly. The model predicts the next 12 months which is the year 2016 because the last available records for sea level anomaly are the year 2015. The value seems less at first glance, but the sea level anomaly scale is very little and sea level is rising at a steady rate. Sea level rose 0.11 to $0.16 \mathrm{~m}$ in the twentieth century [21]. The ARIMA model also accurately identifies and forecasts sea level anomaly. The sea level anomaly is a slow and steady process and often changes gradually. That is why sea level anomaly prediction performs well using the LSTM model.

From the above sections, sudden instance like an earthquake is extremely difficult to predict accurately. There are many factors to be considered when predicting an earthquake. From the previous works, it was found out that predicting earthquake magnitude is almost impossible. Most of the researchers used one-hot encoding to convert earthquake into categorical value and then predict only whether the earthquake will happen or not. In this research, predicting when an earthquake will happen was attempted. The model accurately identifies only until a certain quarter of the year. On the other hand, both ARIMA model and LSTM model performed well for sea level anomaly prediction. This further proves that ARIMA and LSTM model performs well with consistent time-series data.

\section{CONCLUSIONS}

In this paper, endangered countries due to earthquake and sea level rise such as Indonesia was found out and further prediction of an earthquake happening in the future and rising sea level was conducted. It was found out that the LSTM model performed well for predicting both earthquake and sea levels. Using the model earthquake can be predicted accurately until the quarter of the year. Also, for sea level anomaly prediction the model performed accurately.

Earthquake is a sudden calamity that is unpredictable, it is difficult to accurately identify the exact time of occurrence. The process for predicting an event requires consistent history based on which the model will learn and predict accurately. In that case, the sea level anomaly prediction is much easier and effective than predicting the earthquake.

These kinds of research can help understanding earth science much better and find the pattern in natural disasters. This can be transformed and add as addition into an early warning system that can aid the government to prepare better for upcoming natural hazards and relief management tasks. Also, a lot of lives can be saved using these early warning systems.

The approach used in this paper can certainly help to progress in that way. Countries can use the model for sea level anomaly prediction and can see which areas of their country are under risk. Proper measurements and initiatives then can be taken for that area and the government can plan accordingly.

In future, more countries can be added in the model to extend the number of endangered countries such as Japan for earthquakes and Bangladesh for sea level rise. Also, for predicting earthquakes more efficient model can be developed which can predict more accurately in which month the earthquake will happen.

\section{ACKNOWLEDGMENT}

The authors would like to extend the gratitude towards Sheikh Md Hanif Hossain and Ahmad Abu Saiid, and Kulliyyah of Information and Communication Technology (KICT), IIUM.

\section{REFERENCES}

[1] Jakarta Post. (2019). Central Sulawesi disasters killed 4,340 people, final count reveals. Retrieved from https://www.thejakartapost.com/news/2019/01/30/centralsulawesi-disasters-killed-4340-people-final-count-reveals.html.

[2] List of earthquakes in 2018. (2019, October 6). Retrieved from https://en.wikipedia.org/wiki/List of earthquakes in 2018.

[3] Zoological Society of London. (2010, April 1). Toads' e-arthquake exodus. ScienceDaily. Retrieved November 12,2019from www.sciencedaily.com/releases/2010/03/100330210949.htm

[4] K. Godfrey, (2017, November 16). REVEALED: This holiday hotspot is SINKING and will have disappeared within 80 years. Retrieved from

https://www.express.co.uk/travel/articles/879410/maldivesholiday-pacific-islands-sinking-disappear-climate-change

[5] E. Florido, Aznarte, J. L., Morales-Esteban, A., \& MartínezÁlvarez, F. (2016). Earthquake magnitude prediction based on artificial neural networks: A survey. Croatian Operational Research Review, 7(2), 159-169.

[6] K.M.Asim, Martínez-Álvarez, F., Basit, A., \& Iqbal, T. (2017). Earthquake magnitude prediction in Hindukush region using machine learning techniques. Natural Hazards, 85(1), 471-486.

[7] T. Perol, T., Gharbi, M., \& Denolle, M. (2018). Convolutional neural network for earthquake detection and location. Science Advances, 4(2), e1700578.

[8] P.M. DeVries, Viégas, F., Wattenberg, M., \& Meade, B. J. (2018). Deep learning of aftershock patterns following large earthquakes. Nature, 560(7720), 632.

[9] Q. Wang, Guo, Y., Yu, L., \& Li, P. (2017). Earthquake prediction based on spatio-temporal data mining: an LSTM network approach. IEEE Transactions on Emerging Topics in Computing.

[10] G. Asencio-Cortés, Martínez-Álvarez, F., Troncoso, A., \& Morales Esteban, A. (2015). Medium-large earthquake magnitude prediction in Tokyo with artificial neural networks. Neural Computing and Applications, 28(5), 1043-1055.

[11] Z. Li, Meier, M. A., Hauksson, E., Zhan, Z., \& Andrews, J. (2018) Machine learning seismic wave discrimination: Application to earthquake early warning. Geophysical Research Letters, 45(10), 4773-4779.

[12] J.C.B. Gamboa, (2017). Deep learning for time-series analysis. arXiv preprint arXiv:1701.01887. 
[13] M. Imani, You, R. J., \& Kuo, C. Y. (2014). Forecasting Caspian Sea level changes using satellite altimetry data (June 1992December 2013) based on evolutionary support vector regression algorithms and gene expression programming. Global and Planetary Change, 121, 53-63.

[14] A. Braakmann-Folgmann, A., Roscher, R., Wenzel, S., Uebbing B., \& Kusche, J. (2017). Sea Level Anomaly Prediction using Recurrent Neural Networks. arXiv preprint arXiv:1710.07099.

[15] M. Imani, Chen, Y. C., You, R. J., Lan, W. H., Kuo, C. Y., Chang, J. C., \& Rateb, A. (2017). Spatiotemporal Prediction of Satellite Altimetry Sea Level Anomalies in the Tropical Pacific Ocean. IEEE Geoscience and Remote Sensing Letters, 14(7), 1126-1130.

[16] U.S. Geological Survey, 2019, Earthquake Search Catalog, accessed September, 2019 Retrieved from https://earthquake.usgs.gov/earthquakes/search/

[17] ECV products, Climate Change Initiatives, European Space Agency. Retrieved from http://www.esa-sealevelcci.org/products
[18] J.F. Legeais, Ablain, M., Zawadzki, L., Zuo, H., Johannessen, J. A., Scharffenberg, M. G., Fenoglio-Marc, L., Fernandes, M. J., Andersen, O. B., Rudenko, S., Cipollini, P., Quartly, G. D., Passaro, M., Cazenave, A., and Benveniste, J.: An improved and homogeneous altimeter sea level record from the ESA Climate Change Initiative, Earth Syst. Sci. Data, 10, 281-301, https://doi.org/10.5194/essd-10-281-2018, 2018.

[19] World Sea-Level Rise Dataset. (2013, November 20). Retrieved from https://datacatalog.worldbank.org/dataset/world-sealevel-rise-dataset.

[20] GISS, National Aeronautics and Space Administration (NASA), panoply, retrieved from https://www.giss.nasa.gov/tools/panoply/download/

[21] Hay, C. C., Morrow, E., Kopp, R. E. \& Mitrovica, J. X. Probabilistic reanalysis of twentieth-century sea-level rise. Nature 517, 481484 (2015)

[22] Lee, J. (2016, May 20). How Do Earthquake Early Warning Systems Work? Retrieved from https://www.nationalgeographic.com/news/2013/9/130927earthquake-early-warning-system-earth-science/ 